\title{
1 Single Defect Center Scanning Near-Field Optical Microscopy on Graphene
}

2

3

4 J. Tisler, T. Oeckinghaus, R. Stöhr, R. Kolesov, F. Reinhard and J. Wrachtrup

5 3. Institute of Physics, Stuttgart University, 70550 Stuttgart, Germany

\section{Abstract}

10 We demonstrate high resolution scanning fluorescence resonance energy transfer

11 microscopy between a single nitrogen-vacancy center as donor and graphene as acceptor.

12 Images with few nanometer resolution of single and multilayer graphene structures were

13 attained. An energy transfer efficiency of $30 \%$ at distances of $10 \mathrm{~nm}$ between a single

14 defect and graphene was measured. Further the energy transfer distance dependence of

15 the nitrogen-vacancy center to graphene was measured to show the predicted $\mathrm{d}^{-4}$ 16 dependence. Our studies pave the way towards a diamond defect center based versatile 17 single emitter scanning microscope.

\section{Introduction}

20 Imaging the optical near field of nano-sized structures is of fundamental interest to

21 various areas of photonic-, material- and biological sciences.

22 As a result a wealth of scanning near-field optical microscopy (SNOM) techniques are

23 available nowadays ${ }^{1}$. A notable extension to SNOM is scanning fluorescence resonance

24 energy transfer microscopy ${ }^{2}$. The method exploits fluorescence resonance energy 25 transfer $(\text { FRET })^{3}$, a non-radiative dipole-dipole interaction between transition dipole

26 moments of a donor and an acceptor. As the method exploits the near field interaction of 
27 two induced dipoles it has a steep dependence on distance $\mathrm{d}\left(\mathrm{z}^{-6}\right)$ and promises to be

28 capable of achieving $\mathrm{nm}$ resolution. Different donors and acceptor systems have been

29 used like dyes ${ }^{4}$, color centers ${ }^{5}$ or quantum $\operatorname{dots}^{6}$. But most of these systems suffer from

30 blinking or limited photostability rendering their use cumbersome in practice At low

31 temperature single molecular fluorescence usually is stable and as a consequence

32 scanning fluorescence imaging of light fields has been demonstrated ${ }^{7}$. One particular

33 photoactive system, namely the nitrogen-vacancy (NV) center in diamond has gained

34 attention as an atom-like photon emitter. It proves to be photostable even at room

35 temperature ${ }^{8}$, can be brought into nm proximity of any photonic system ${ }^{9}$ and has been

36 proposed as a stable emitter for near-field microscopy ${ }^{4}$. As a result FRET has been

37 demonstrated between a NV and another single molecule with high transfer efficiency

$38{ }^{10,11}$.Therefore it appears to be an ideal emitter for FRET SNOM. In this study we utilize

39 a scanning near field FRET microscope to investigate the interaction of a single NV

40 emitter with graphene. Graphene has a number of intriguing near-field optical properties

41 like high mode density ${ }^{12}$ and it is speculated that graphene plasmons are easily lauched

42 by near field interactions with emitters. Further on only $2.3 \%$ of the incoming far field

43 plane wave absorbed from a monolayer ${ }^{13}$ making near and far field effects to be clearly

44 distinguishable. Owing to its two dimensional geometry the distance dependence of the

45 FRET efficiency is expected to be $\mathrm{z}^{-4}$ dependent ${ }^{14}$ rather that $\mathrm{z}^{-6}$. Additionally the FRET

46 transfer efficiency probes relevant material properties like the local fermi energy.

49 Results 
50 All the experiment use a home-build scanning FRET microscope based on an atomic

51 force microscope (AFM) with an optically accessible tip. Single NV centers inside

52 nanodiamonds on the tip of the AFM are used as FRET donors. A laser beam exciting the

$53 \mathrm{NV}$ center is focused through the sample onto the NV. Fluorescence of the defect center

54 is collected through the same channel (see Fig. 1).

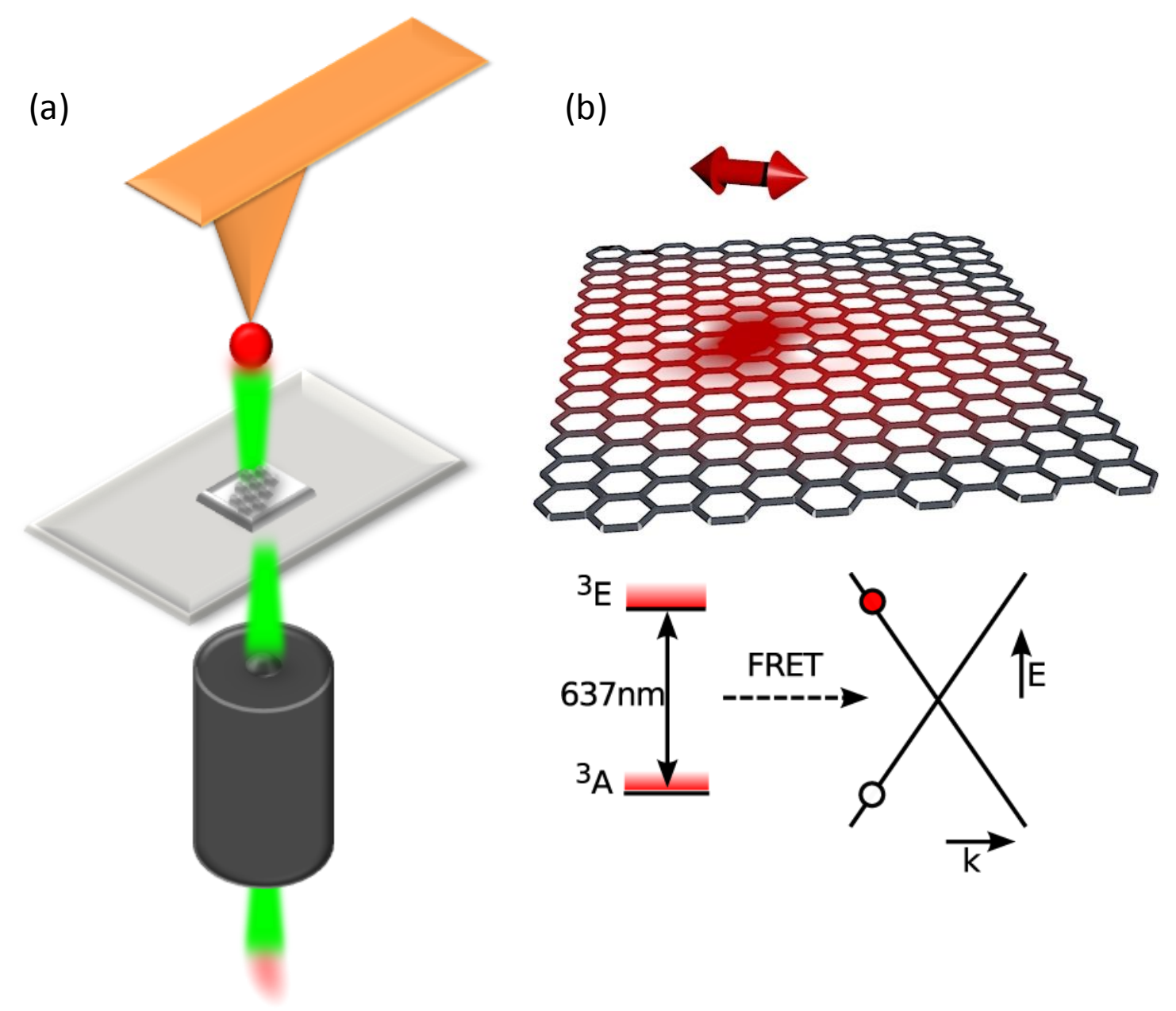

56 Figure 1: Schematic images of the experiment (a) Schematic image of the

57 experimental setup. Nanodiamonds are glued onto the apex of a Si tip. A high

58 numerical aperture objective (NA 1.3 is used to excite a single $\mathrm{NV}$ in the

59 nanodiamond and collect NV fluorescence. (b) Energy levels of the lowest optical 
60 transition relevant for energy transfer and and graphene band structure near the $\Gamma$

61 point.

62

63 To allow for close proximity between the defect and graphene small nanodiamond with

64 diameters around $\sim 25 \mathrm{~nm}$ containing single NV centers were used. When the NV center

65 is placed in close proximity to the graphene sample, its fluorescence is quenched by

66 Förster energy transfer. In this process, an electronic excitation of the NV center is

67 nonradiatively transferred into an exciton in graphene (Fig. 1b) which quickly dissipates

68 excitation energy mostly by internal radiationless decay ${ }^{15}$ ccuring at a rate $\gamma_{n r}$, this

69 process competes with radiative emission of the NV center and reduces the fluorescence

70 intensity to a value

$$
I=\frac{\gamma_{e x} \gamma_{r}}{\gamma_{r}+\gamma_{n r}}
$$

71 where $\gamma_{e x}$ and $\gamma_{r}$ are the excitation rate and radiative rate, respectively, and we assume

$72 \gamma_{e x}$ to be much below saturation.

73 The quenching rate $\gamma_{n r}$ can be computed from Förster's law ${ }^{16}$ with the additional

74 assumption that a sheet of graphene can be approximated by a two-dimensional array of

75 infinitesimal flakes ${ }^{17}$.

$$
\gamma_{n r}=A \iint_{\substack{\text { graphene } \\ \text { sheet }}} d s^{2}\left|\boldsymbol{E}_{\boldsymbol{p}}\right|^{2} \mu_{g}{ }^{2}=A \iint_{\substack{\text { graphene } \\ \text { sheet }}} d s^{2} \frac{\mu_{e g}{ }^{2} \mu_{g}{ }^{2} f\left(\widehat{\boldsymbol{r}}, \widehat{\boldsymbol{\mu}_{\boldsymbol{e g}}}\right)}{r^{6}}
$$

76 Here, A is a proportionality constant and $\boldsymbol{E}_{\boldsymbol{p}}=\boldsymbol{E}-\left(\boldsymbol{e}_{\boldsymbol{z}} \cdot \boldsymbol{E}\right) \boldsymbol{e}_{\boldsymbol{z}}$ denotes the in-plane

77 component of $\boldsymbol{E}=\left(3 \hat{\boldsymbol{r}}\left(\boldsymbol{\mu}_{\boldsymbol{e g}} \cdot \hat{\boldsymbol{r}}\right)-\boldsymbol{\mu}_{\boldsymbol{e g}}\right) /\left(4 \pi \epsilon_{0} r^{3}\right)$, the near-field of the transition

78 dipole $\boldsymbol{\mu}_{\boldsymbol{e g}}=-e\langle e|\boldsymbol{r}| g\rangle$ of the NV center's optical transition between states $|g\rangle$ and $|e\rangle$

79 (red shade in Fig. 1b). The graphene transition dipole moment $\mu_{g}$ is a scalar, reflecting the 
80 fact that graphene is an isotropic material. Hence, exciton transitions can be driven by

81 any in-plane driving field $\boldsymbol{E}_{\boldsymbol{p}}=E \boldsymbol{e}_{\boldsymbol{p}}$, regardless of its polarization $\boldsymbol{e}_{\boldsymbol{p}}$. Precisely,

$82 d s^{2} \mu_{g}{ }^{2}=e^{2} \sum_{\boldsymbol{k}_{\boldsymbol{i}}, \boldsymbol{k}_{\boldsymbol{f}}, \omega=v_{F}\left(\boldsymbol{k}_{\boldsymbol{f}}+\boldsymbol{k}_{\boldsymbol{i}}\right)}\left|\left\langle\boldsymbol{k}_{\boldsymbol{f}}|x| \boldsymbol{k}_{\boldsymbol{i}}\right\rangle\right|^{2}$ where $\quad\left|\boldsymbol{k}_{\boldsymbol{i}}\right\rangle,\left|\boldsymbol{k}_{\boldsymbol{f}}\right\rangle$ denote plane-wave

83 electron states in graphene and $\omega$ is the NV transition's frequency.

84 Integrating equation (1) over an infinitely large graphene surface yields a modified

85 Förster type law

$$
\gamma_{n r}=\gamma_{r} \frac{z_{0}^{4}}{z^{4}}
$$

86 with a Förster distance $z_{0}$ and a quenching rate rising with the fourth power of distance,

87 differing markedly from the $z^{-6}$ law commonly known for point like objects such as

88 molecules or atoms.

89 Quantitatively, the Förster distance can be calculated from eq. (2) and the expressions of

$90 \quad \gamma_{n r}$ and $\gamma_{r}$ stated in previous work ${ }^{714}$.

91

$$
\mathrm{z}_{0}=\sqrt[4]{\frac{9 \mathrm{e}^{2} \hbar^{3} \mathrm{c}^{3}}{4 \cdot 512(\hbar \omega)^{4} \epsilon_{0}}}=15.3 \mathrm{~nm}
$$


(a)

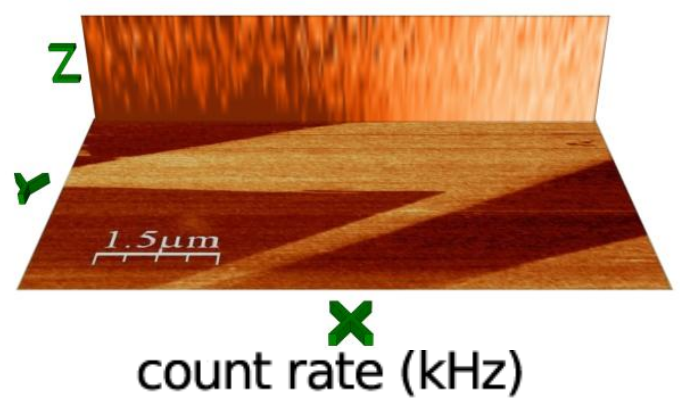

(b)
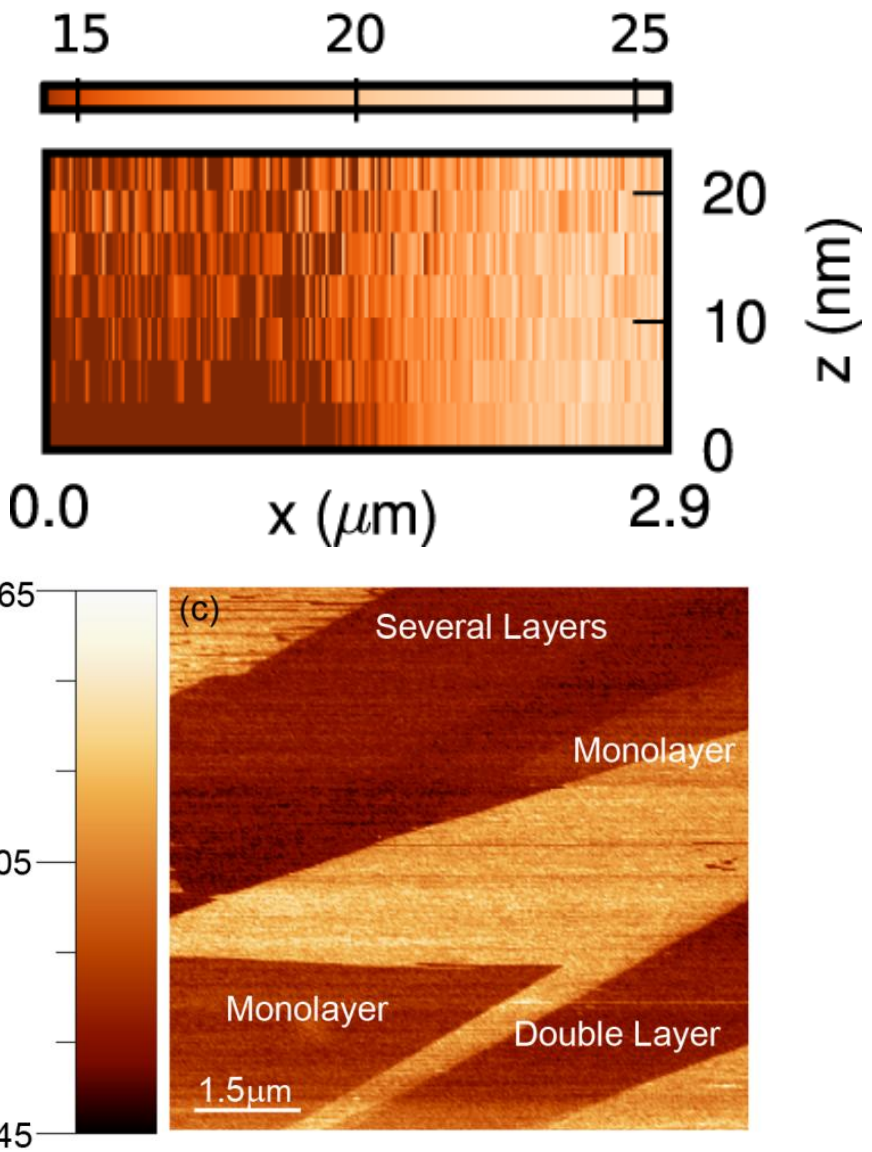

97 Figure 2: Scanning FRET on graphene mono- and multiple layers. Plots show the

98 NV fluorescence intensity as a function of lateral (c) as well as lateral and axial

99 position over the graphene sample.

100 In the following we discuss our scanning FRET experiments on layers of different

101 graphene thickness. It is worth mentioning that in addition to lateral xy scans we also 
102 measured in $\mathrm{xz}-$ and in xy-direction. For such images tapping mode scanning FRET is

103 used. In this mode the tip of the AFM is oscillating during scanning in contrast to contact

104 mode. The photons arriving during the scan are measured in registry with the different

105 heights of the oscillating tip.. Fig. 2b shows a corresponding image in the xz plane. To

106 compare eq. (1) with results from Fig. 2c, we transform the fluorescence

$107 I(x, y, z)$ observed in measurements into a quenching rate $\gamma_{n r}(x, y, z)$ by the relation

$$
\gamma_{n r}=\left(\frac{I_{0}}{I(x, y, z)}-1\right) \gamma_{r},(4)
$$

109 where $I_{0}$ is the unquenched NV fluorescence intensity. In all the following, we assume a

110 radiative rate $\gamma_{r}=1 / \tau_{N V}$ based on the measured fluorescence lifetime $\tau_{N V}=13 \mathrm{~ns}^{18}$.

111 Note that this conversion implicitly assumes unity quantum efficiency.

112 In case of Fig. 2 the monolayer quenching rate was $27.7 \mathrm{E}^{6} \mathrm{~s}^{-1}$ for a double layer $33.1 \mathrm{E}^{6} \mathrm{~s}^{-1}$

113 and for several layers $46.2 \mathrm{E}^{6} \mathrm{~s}^{-1}$. In contrast to previous work ${ }^{19}$ no exact scaling with

114 layer thickness was observed, probably due to background contributions which were not

115 taken into account in our measurements. 

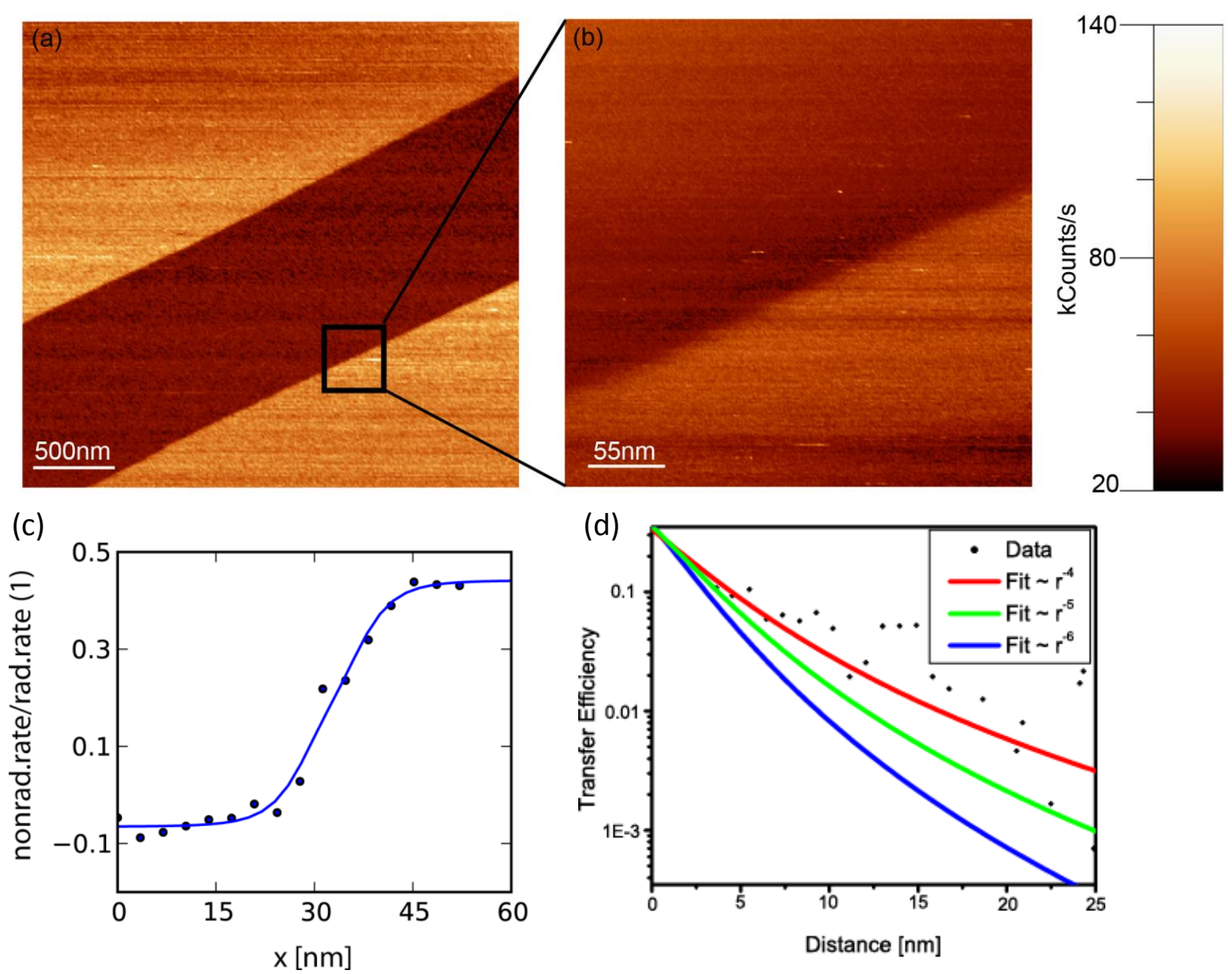

117 Figure 3: Quantitative comparison of scanning fluorescence resonance energy

118 transfer microscopy images to theory. A high resolution contact-mode scan of a

119 graphene edge (a-b) is used to measure the step response function of the single NV

120 center scanning over the graphene monolayer (c) The solid line of (c) is a fit to the

121 data using eq. (1), parametrized by the $\mathbf{N V}$-graphene distance of $z_{N V}=9.8 \mathrm{~nm}$. (d)

122 Vertical dependence $\gamma_{N V}(z)$. A fit of the data (red line) agrees with the predicted

$123 z^{-4}$ law (eq. (2)) for a Förster distance of $z_{0}=8.25 \pm 0.13 \mathrm{~nm}$.

125 Thanks to their atomic size, scanning single emitters are able to map near-field couplings

126 with molecular $(\mathrm{nm})$ resolution in all three dimensions. As a first application of this 
127 remarkable property, we experimentally confirmed the theoretical model of eq. (1). The

128 result is shown in Fig. 3. In the lateral dimensions (Fig. 3 a-c), a high-resolution scan of a

129 graphene edge (Fig. 3b) reveals that fluorescence drops smoothly over a length scale of

$130 \sim 10 \mathrm{~nm}$ when the NV center is moved over the flake. The theoretical prediction (solid line

131 in Fig. 3c) is obtained by numerically integrating equation (1), taking into account the

132 fact that the NV transition has two orthogonal transition dipoles $\boldsymbol{\mu}_{\mathbf{1}}, \boldsymbol{\mu}_{\mathbf{2}}$

$$
\begin{gathered}
\gamma_{n r}(x)=\left(\gamma_{n r}^{\boldsymbol{\mu}_{1}}(x)+\gamma_{n r}^{\boldsymbol{\mu}_{2}}(x)\right) / 2 \\
\gamma_{n r}^{\boldsymbol{\mu}}(x)=A \mu_{g}{ }^{2} \int_{x}^{\infty} d x \int_{-\infty}^{\infty} d y\left|\boldsymbol{E}_{\boldsymbol{p}}{ }^{{ }^{z_{N V}, \boldsymbol{\mu}}}(x, y)\right|^{2} .
\end{gathered}
$$

133 The remaining free parameters $A, \mu_{g}{ }^{2}$ and $Z_{N V}$ are fit to the data and the dipole 134 orientation is chosen as $\boldsymbol{\mu}_{\mathbf{1}}\left\|\boldsymbol{e}_{z}, \boldsymbol{\mu}_{\mathbf{2}}\right\| \boldsymbol{e}_{\boldsymbol{x}}$ to best fit the observed bahaviour. We find that 135 the data agrees well with the theoretical prediction (Fig. 3c). The achieved resolution is 136 limited by the spatial extent of the near-field $\boldsymbol{E}_{\boldsymbol{p}}$, which is of the order of the NV137 graphene distance. Reverting the argument, we can extract this distance from the fit, 138 finding that $z_{N V}=9.8 \mathrm{~nm}$. This value is nonzero even though the nanodiamond was 139 scanned over the graphene in contact mode. Therefore, we interpret this value as the 140 distance between the NV center and the surface of the nanodiamond.

141 We also measured the vertical dependence $\gamma_{n r}(z)$ by repeatedly approaching and 142 retracting the tip on a large graphene surface (Fig 3d). Again, we find that the data is well 143 described by eq. (1) and in particular agrees with the predicted $z^{-4}$ law of eq. (2). Using 144 the value $z_{N V}$ obtained from the lateral scan, we can quantitatively fit the data and infer a 145 Förster distance of $z_{0}=8.25 \pm 0.13 \mathrm{~nm}$. This differs significantly from the theory 146 prediction $\left(z_{0}=15.3 \mathrm{~nm}\right.$, eq. (3)). Most likely, this difference is due to additional 
147 nonradiative channels which lower the NV center's quantum efficiency and thus reduce

$148 z_{0}$. The existence of such channels is likely and candidates include quenching by the

149 silicon AFM tip or an intrinsically low quantum efficiency in nanodiamonds. Conversely,

150 we note that our result provides a method to measure the quantum efficiency of a single

151 emitter when it is combined with a quantitative theory of excitonic transitions in 152 graphene ${ }^{1420}$.

\section{Conclusion}

155 We demonstrated optical scanning fluorescence resonance energy transfer microscopy 156 with a single NV center in nanodiamond as emitter. By scanning over a graphene

157 monolayer we attained nanometer resolution images and a transfer efficiency as large as

$15830 \%$. Furthermore we experimentally confirmed a $z^{-4}$ dependence of the energy transfer

159 rate between a point-like emitter and a graphene monolayer. While graphene is an

160 interesting photonic material in its own right, application of the technique to other nano

161 photonic structures and acceptors, e.g. single molecules certainly would be of great

162 interest. Applications in biological sciences where scanning FRET might become a

163 valuable addition to other FRET based techniques for, e.g. imaging larger protein

164 structures of cellular surfaces are easily envisioned. Such methods may be combined with

165 the magnetic field sensing capabilities of the NV center to yield a truly multifunctional 166 local probe. 
170 Financial support by the EU via projects SQUTEC and DINAMO as well as the German

171 Science foundation via research group 1493 and SFB/TR 21 is acknowledged.

\section{References}

174 1. Betzig, E., Trautman, J. K., Harris, T. D., Weiner, J. S. \& Kostelak, R. L. Breaking

175 the Diffraction Barrier: Optical Microscopy on a Nanometric Scale. Science 251,

$176 \quad 1468-1470$ (1991).

177 2. Sekatskii, S. K. \& Letokhov, V. S. Nanometer-resolution scanning optical microscope with resonance excitation of the fluorescence of the samples from a single-atom excited center. Jetp Lett. 63, 319-323 (1996).

3. Lakowicz, J. R. Principles of fluorescence spectroscopy. (Springer: 2006).

4. Gruber, A. et al. Scanning Confocal Optical Microscopy and Magnetic Resonance on Single Defect Centers. Science 276, 2012-2014 (1997).

5. Shubeita, G. T. et al. Scanning near-field optical microscopy using semiconductor nanocrystals as a local fluorescence and fluorescence resonance energy transfer source. J Microsc 210, 274-278 (2003).

6. Y. Ebenstein, T. M. Quantum-Dot-Functionalized Scanning Probes for FluorescenceEnergy-Transfer-Based Microscopy. (2003).doi:10.1021/jp036135j

7. Michaelis, J. et al. A single molecule as a probe of optical intensity distribution. Opt. Lett. 24, 581-583 (1999).

8. Michalet, X. et al. Quantum Dots for Live Cells, in Vivo Imaging, and Diagnostics. Science 307, 538 -544 (2005).

9. Kurtsiefer, C., Mayer, S., Zarda, P. \& Weinfurter, H. Stable Solid-State Source of Single Photons. Phys. Rev. Lett. 85, 290-293 (2000).

10. Tisler, J. et al. Highly Efficient FRET from a Single Nitrogen-Vacancy Center in Nanodiamonds to a Single Organic Molecule. ACS Nano 5, 7893-7898 (2011).

11. Kühn, S., Hettich, C., Schmitt, C., Poizat, J.-P. \& Sandoghdar, V. Diamond colour centres as a nanoscopic light source for scanning near-field optical microscopy. Journal of Microscopy 202, 2-6 (2001).

12. Koppens, F. H. L., Chang, D. E. \& García de Abajo, F. J. Graphene Plasmonics: A Platform for Strong Light-Matter Interactions. Nano Letters (2011).at <http://resolver.caltech.edu/CaltechAUTHORS:20110929-105946388>

13. Nair, R. R. et al. Fine Structure Constant Defines Visual Transparency of Graphene. Science 320, 1308-1308 (2008).

14. Swathi, R. S. \& Sebastian, K. L. Long range resonance energy transfer from a dye molecule to graphene has (distance) -4 dependence. The Journal of Chemical Physics 130, 086101-086101-3 (2009). 
15. Stöhr, R. J., Kolesov, R., Pflaum, J. \& Wrachtrup, J. Fluorescence of laser-created electron-hole plasma in graphene. Phys. Rev. B 82, 121408 (2010).

16. Förster, T. Zwischenmolekulare Energiewanderung und Fluoreszenz. Annalen der Physik 437, 55-75 (1948).

17. Stöhr, R. J. et al. Super-resolution Fluorescence Quenching Microscopy of Graphene. ACS Nano 6, 9175-9181 (2012).

18. Robledo, L., Bernien, H., Sar, T. van der \& Hanson, R. Spin dynamics in the optical cycle of single nitrogen-vacancy centres in diamond. New Journal of Physics 13, 025013 (2011).

19. Chen, Z., Berciaud, S., Nuckolls, C., Heinz, T. F. \& Brus, L. E. Energy Transfer from Individual Semiconductor Nanocrystals to Graphene. ACS Nano 4, 2964-2968 (2010).

20. Swathi, R. S. \& Sebastian, K. L. Resonance energy transfer from a dye molecule to graphene. The Journal of Chemical Physics 129, 054703 (2008). 